\title{
Preoperative Prediction of Difficulty of Laparoscopic Cholecystectomy
}

\author{
Hanna Habib Hanna, Mohamed Ibrahim Mohamed and Amir Milad Nazier* \\ Department of General Surgery, Faculty of Medicine, Ain Shams University \\ *Corresponding Author: Amir Milad Nazier, Phone No.: (+2) 01212653453, E-mail: amir-1001@ @otmail.com
}

\begin{abstract}
Background: laparoscopic cholecystectomy (LC), one of the most commonly performed surgical procedures worldwide, is accepted as the gold standard in the treatment of symptomatic gallstones for its minimal invasiveness, less pain and early recovery.
\end{abstract}

Purpose: to predict the difficulty of laparoscopic cholecystectomy in patients according to the recently published scoring system and to add more items to it.

Patients and Methods: this is a prospective cohort study. This study took place in Ain Shams University Hospital and Manshiet El Bakry Public Hospital, General Surgery Unit, Surgery Department; the study involved 120 patients admitted with calcular cholecystitis, arranged for laparoscopic cholecystectomy.

Results: in our study we found that age, sex and ultrasonographic data were significant predictive factors for assessment preoperatively difficult cases that will be operated upon. We found 14 patients above 50 years who scored to be difficult and very difficult were at outcome difficult, only three patients converted to open surgery over fifty.

Conclusion: According to sex males has been described to be associated with difficult LC as in our study that confirmed that as 14 males who participated in our study 10 of them were predicted to have a difficult surgery and 3 expected to be very difficult. Post-surgery $100 \%$ of males turned out to have a difficult procedure. Also according to other factors, such as history of acute attacks that increase risk and difficulty due to adhesions at Calot's triangle and risk of cystic artery and bile spillage, were increased during dissection.

Keywords: Laparoscopic Cholecystectomy - Acute Cholecystitis - Pancreatic Duct

\section{INTRODUCTION}

Laparoscopic cholecystectomy, one of the most commonly performed surgical procedures worldwide, is accepted as the gold standard in the treatment of symptomatic gallstones for its minimal invasiveness, less pain and early recovery ${ }^{(1)}$.

Although laparoscopic cholecystectomy has generally a low incidence of morbidity and mortality and of conversion rate to open surgery, its outcome is particularly affected by the presence and severity of inflammation, advancing patients' age, male sex and greater body mass index ${ }^{(2)}$.

Sometimes

laparoscopic cholecystectomy becomes difficult. It takes longer time even with bile/stone spillage and occasionally it requires conversion to open cholecystectomy ${ }^{(3)}$.

It may be difficult to anticipate preoperatively whether this procedure is going to be easy or difficult in a particular patient.
The degree of difficulties is again impossible to predict but it is important to know for better preparedness for the surgeon and explanation to patients for possibility of conversion to open ${ }^{(3)}$

Preoperative assessment of complexity factors is needed for frequent procedures such as laparoscopic cholecystectomy in order to avoid complications and delays and to guarantee an efficient course of surgery ${ }^{(4)}$.

Previous upper abdominal surgery is associated with a higher rate of adhesions, an increased risk of operative complications, a greater conversion to open surgery rate, a prolonged operating time and longer hospital stay. Also, laparoscopic cholecystectomy after endoscopic retrograde cholangiopancreatography (ERCP) with endoscopic sphincterotomy (ES) for combined choledochocystolithiasis is more difficult with prolonged procedure than in uncomplicated 
gallstone disease with a longer post-operative hospital stay ${ }^{(5)}$.

Preoperative complexity estimation in laparoscopic cholecystectomy helps surgeons decide whether to proceed with a minimally invasive approach, perform an open procedure or make a referral to a more experienced surgeon. It may also be useful for explaining the various risks of laparoscopic and open procedures ${ }^{(6)}$.

In this study, we will assess the difficulty of laparoscopic cholecystectomy preoperatively and predict its difficulty according to some factors and items.

\section{PATIENTS AND METHODS}

- Type of Study: Prospective cohort study.

- Study Setting:

The study was conducted in the General Surgery Department in Ain Shams University Hospitals, and in the General Surgery Department in Manshiet El Bakry Public Hospital.

\section{- Study Period}

The study was conducted from Jan 2018 to June 2018.

\section{Study Population}

Patients who came to the outpatient clinics and ER of Surgery Department at Ain Shams University Hospitals and to Manshiet El Bakry Public Hospital, presented with calcular cholecystitis from Jan 2018 to June 2018.

\section{- Inclusion Criteria:}

- Patients' age ranged from 20 to 65 years old.

- Patients from both gender.

- Patients presenting to the General Surgery Department in Ain Shams University Hospitals, and to the General Surgery Department in Manshiet El Bakry Public Hospital with calcular cholecystitis.

\section{- Exclusion Criteria:}

- Conversion to open cholecystectomy due to equipment failure.

- Prolonged operation time due to other causes not related to patients as anesthesia and junior surgeon while training.

- Sampling Method: Simple random sample

\section{- Sample Size:}

120 persons were randomly selected from patients presented to Ain Shams University Hospitals and Manshiet El Bakry Public
Hospital presented with biliary colic for laparoscopic cholecystectomy

- Ethical Considerations

1. Data were collected after permission of the responsible authorities.

2. Author introduced himself to the patients and explained the aim, the benefits and the hazards of the procedures which were performed for each patient before getting them involved in the study.

3. Data were collected from the patients after taking their informed consent.

4. Collected data were kept as a confidential data and were used only for research purposes.

5. Any patient refuses to participate in the study was not enrolled in the study

6. Patients had the right to withdraw from the study at any time without giving reasons, and this didn't affect the medical care expected to be offered to them. The study was approved by the Ethics Board of Ain Shams University.

\section{- Statistical Analysis:}

Data collected throughout history, clinical examination, laboratory investigations and outcome measures were coded, entered and analyzed using Microsoft Excel software. Data then were imported into Statistical Package for the Social Sciences (SPSS version 11.0) software for analysis. Chi-square test was used to test the significance of relations between different variables. $\mathrm{P}$ value $<0.05$ was considered significant.

\section{RESULTS}

Table (1): Distribution of study population according to conversion

\begin{tabular}{|l|c|c|}
\hline $\begin{array}{c}\text { Conversion to open } \\
\text { surgery }\end{array}$ & Number & $\begin{array}{c}\text { Percentage } \\
(\%)\end{array}$ \\
\hline No & 109 & $90.8 \%$ \\
\hline Yes & 11 & $9.2 \%$ \\
\hline Total & 120 & $100 \%$ \\
\hline
\end{tabular}

This table showed rate of conversions of patients from LC to open surgery and their percentage.

Table (2): Correlation of preoperative score and the outcome

\begin{tabular}{|c|c|c|c|}
\hline \multirow{2}{*}{$\begin{array}{c}\text { Preoperative } \\
\text { scoring parameter }\end{array}$} & \multicolumn{3}{|c|}{ Outcome } \\
\hline & Easy & Difficult & Total \\
\hline Easy & $\begin{array}{c}54 \\
(93.1 \%)\end{array}$ & $\begin{array}{c}29 \\
(46.8 \%)\end{array}$ & $\begin{array}{c}83 \\
(69.2 \%)\end{array}$ \\
\hline Difficult & $4(6.9 \%)$ & $\begin{array}{c}29 \\
(46.8 \%) \\
\end{array}$ & $\begin{array}{c}33 \\
(27.5 \%) \\
\end{array}$ \\
\hline V. Difficult & $0(0 \%)$ & $4(6.4 \%)$ & $4(3.3 \%)$ \\
\hline Total & 58 & 62 & 120 \\
\hline
\end{tabular}


This table showed correlation of preoperative score and the outcome intraoperative and postoperative which define that all patients expected to be difficult preoperative by scoring system were actually difficult intraoperative due to predefined factors detected at scoring system.

14 patients above fifty who scored to be difficult and very difficult were at outcome scoring difficult $(100 \%$ of cases) that's mean significant correlation between age and the difficult level of surgery.

In our study, there were 14 males and 106 females. Of 14 males, 10 were predicted to have a difficult surgery and 3 expected to be very difficult. Post-surgery $100 \%$ of males turned out to have a difficult procedure.

There were 11 patients had history of admission to hospital due to repeated attacks of acute cholecystitis, all 11 (100\%) patients were predicted to be difficult and intraoperative outcome was difficult.

102 patients were $\mathrm{BMI} \geq 25$ only 33 predicted to be difficult and outcome was 58 patients had difficult operation.

10 patients had clinically palpable GB and out of them $100 \%$ turned out to have a difficult procedure.

Upper abdominal scars (indicators of previous upper abdominal surgeries) may lead to intraperitoneal adhesions that cause increased probability of injury and bleeding while placement of umbilical port. It was found to be statistically significant factor in our study as 8 of 55 patients had previous scars had upper abdominal scars $75 \%$ of them converted to open due to severe adhesions.

$12.5 \%$ of patients (15 out of 120 ) in our series had GB stone impacted at the neck of GB and turned out to be difficult. It was found to be a statistically significant factor in predicting the difficulty of the procedure in our study. (100\%) predicted to be difficult and $100 \%$ of them were intraoperative difficult due to distension of GB and thick GB wall.

In our study, we found no significant correlation between the GB wall thickness and the difficulty level of surgery (65 of 120) had wall thickness $>4 \mathrm{~mm}, 32$ patients (about $50 \%$ ) of them predicted to be difficult and the 10 cases who were converted to open cholecystectomy were had thick wall of GB.

Pericholecystic collection was found to be a predictor of difficult LC. Postoperatively we found $100 \%$ of these patients (22) out of (22) having difficult LC. We found also that the 10 cases who were converted to open cholecystectomy had pericholecystic collection surrounding GB. Hence, we found a strong correlation between pericholecystic collection and difficult LC.

The overall conversion rate in our study was $8.3 \%$, all ten cases had mostly similar predictive factors such as palpable GB, pericholecystic collection, increased wall thickness of GB and BMI $\geq 25,6$ cases had upper abdominal surgery before, sex and age factors showed no significance for conversion rate.

Intraoperative Factors that increase the difficulty of LC (Bile spillage, bleeding from cystic artery and prolonged time of operation) were mostly depending on predefined factors detected such as: pericholecystic collection, palpable gall bladder, previous upper abdominal operations, impacted stones and increased GB wall thickness. At our study there were cystic artery injury with 25 cases, bile spillage with 33 cases and 55 cases suffered from prolonged time of operation; all previous intraoperative data increase the risk of difficulty and rate of conversion.

\section{DISCUSSION}

Laparoscopic cholecystectomy (LC) is the gold standard treatment of symptomatic cholelithiasis. It is important to predict difficult LC preoperatively so that senior surgeons can be requested to be present during surgery rather than less experienced junior surgeon, which may lead to prolonging the surgery which may lead to intraoperative complications. ${ }^{(7)}$

In preoperatively predicted to be difficult, early decision of conversion can be made so as to avoid unnecessarily prolonging the surgery and to prevent complications. Many studies have attempted form a scoring system to predict difficult LC, but most of them are complex, use large number of determining factors, and they are difficult to use in day to day practice and many of these scoring systems cannot be applied preoperatively. ${ }^{(7)}$

In our study laparoscopic cholecystectomy was performed in 120 patients and different predictive risk factors for difficult laparoscopic cholecystectomy were analyzed. Old age, male sex, history of 
hospitalization, obesity, previous abdominal surgery, palpable gall bladder, and ultrasonographic findings like gall bladder wall thickness, pericholecystic fluid collection, impacted stone were included as risk factors in this study.

Old age (age > 50 years) has been found to be a significant risk factor for difficult laparoscopic cholecystectomy in many studies. In our study, the majority of patients were in the age group of $\leq 50$ (80 patients) and $33.3 \%$ (40 patients) were $>50$ years. In our study we found that 14 patients above fifty who scored to be difficult and very difficult were at outcome scoring difficult $(100 \%$ of cases) that's mean significant correlation between age and the difficult level of surgery, only 3 patients converted to open surgery over fifty, while patients less than 50 years and predicted to be difficult were 18 of 80 patients at this category showed that patients less than fifty were easier than over fifty. ${ }^{(7)}$

Higher conversion rate had been reported in old age group patients ${ }^{(7)}$ but in our study may be due to distribution of cases in our study there is no significant rate of conversion related to old age.

Worldwide, male sex has been described to be associated with difficult LC. ${ }^{(8)}$

Other studies with large sample number has no significant relation between sex and difficulty similar to results by Chndio et $\boldsymbol{a l}$. but this is in contrast with many studies and literatures showed significant association. ${ }^{(9)}$

In the present study, there were 14 males and 106 females. Of 14 males, 10 were predicted to have a difficult surgery and 3 expected to be very difficult. Post-surgery $100 \%$ of males turned out to have a difficult procedure. In our study, there was statistically significance in the relation between male sex and difficulty of LC. Unequal distribution of patients on the basis of sex could have altered the results in the study

Conversion rate and significantly higher mortality has been reported in male sex. (9) Only three males converted to open cholecystectomy due to un-controllable bleeding from cystic artery and bile and stone spillage intraperitoneal.

At this study there were 11 patients had history of admission to hospital due to repeated attacks of acute cholecystitis, all 11 (100\%) patients were predicted to be difficult and intraoperative bile spillage made the operation longer than expected time and outcome were difficult, there were significant relation between history of acute attack and difficulty.

Patients who require hospitalization for repeated attacks of acute cholecystitis carry more chances of difficult laparoscopic cholecystectomy and conversion, probably due to dense adhesions at Calot's triangle and gall bladder fossa. There are reports of higher rate of bleeding, ductal injury and subsequent conversion in acute cholecystitis. ${ }^{(\mathbf{1 0})}$

Obese patients may have a difficult laparoscopic surgery due to various factors; port placement in obese patient takes longer time due to the thick abdominal wall, dissection at the Calot's triangle is also technically difficult due to the obscure anatomy because of excessive intraperitoneal fat and difficulty in the manipulation of instruments through an excessively thick abdominal wall. ${ }^{(\mathbf{1 1})}$

In our study 102 patients were BMI $\geq$ 25 only 33 predicted to be difficult and outcome was 58 patients had difficult operation, surgical expertise of the operating surgeon could be one of the reasons for this discrepancy. BMI was not found to be a predictor ( $\mathrm{p}$ 0.136) according to outcome score of difficult cholecystectomy.

Chang et al. (12) also studied the impact of body mass index on laparoscopic cholecystectomy in Taiwan. Based on their results, they opined BMI was not associated with clinical outcomes and that LC is a safe procedure in obese patients with uncomplicated gallstone disease and laparoscopic surgery has been suggested by some as the preferred approach for obese patients. ${ }^{(13)}$

One of clinical parameters of assessment of difficult LC was palpable GB, it was found to be predictor of difficult LC. Palpable GB could be due to a distended GB, mucocele GB, thick-walled, or due to the adhesions between the GB and the omentum. (13)

In our study, only 10 patients had clinically palpable GB and out of them $100 \%$ (10 of 10) turned out to have a difficult procedure post-surgery and three of them converted to open surgery.

After previous upper or lower abdominal surgery there may be adhesions 
present between viscera or omentum and abdominal wall. There may be chances of injury to these structures during insertion of first port and risk of conversion was reported to be higher. ${ }^{(14)}$

While performing LC, stone impacted at the neck of GB poses some technical problems, because of distension of GB, as is with thick GB wall. It is difficult to grasp the GB neck to allow adequate retraction to perform dissection at the Calot's triangle. ${ }^{(14)}$

Increased GB wall thickness is associated with difficult dissection of the GB from its bed. Presence of a thick GB wall may make grasping and manipulation of GB difficult. This makes the dissection at the Calot's triangle and the GB bed to be difficult and limits the extent of anatomical definition. Thickened gall bladder wall is an ultrasonographic finding of acute cholecystitis and it was a significant factor in previous studies. ${ }^{(15)}$

Baki in 2006 showed that a preoperative gallbladder ultrasound evaluation for symptomatic cholecystitis, which documents a thick gallbladder wall $(\geq 3 \mathrm{~mm})$ with calculi, is a clinical warning for the laparoscopic surgeon of the potential for a difficult laparoscopic cholecystectomy procedure which may require conversion to an open cholecystectomy procedure. ${ }^{(14)}$

Gabriel et al. conducted a study which included 536 patients who underwent laparoscopic cholecystectomy the overall conversion rate in their study was $7.81 \%$. ${ }^{(10)}$ Sharma et al. conducted a study on 200 patients undergoing laparoscopic cholecystectomy at Kathmandu medical college, the conversion rate in their study was $4 \%$. ${ }^{(17)}$

\section{CONCLUSION}

- According to sex males has been described to be associated with difficult $\mathrm{LC}$ as in our study that confirmed that as 14 males who participated in our study 10 of them were predicted to have a difficult surgery and 3 expected to be very difficult. Post-surgery $100 \%$ of males turned out to have a difficult procedure, Also according to other factors such as history of Acute attacks that increase risk and difficulty due to adhesions at Calot's triangle and risk of cystic artery and bile spillage were increased during dissection.
- Conversion rate was $8.3 \%$ (10 of 120 patients) occurred and they had following risk factors (BMI > $25 \mathrm{Kg} / \mathrm{M} 2$, scars of previous abdominal operations, palpable GB, increased wall thickness and pericholecystic collection)

- Another noted data found that complications such as bleeding from cystic artery or bile spillage if occurred intraoperatively increased risk of conversion to open surgery or made the operation more difficult.

- In conclusion we can report that obese patient who were over fifty with history of previous upper abdominal surgery and ultrasonographic picture showed thick walled GB and pericholecystic collection had high risk of conversion.

- At this study scoring system was used for prediction of difficult laparoscopic cholecystectomy sensitivity was $93.75 \%$ and specificity was $52.94 \%$ of the scoring system at score 5 for prediction of easy or difficult laparoscopic cholecystectomy.

\section{REFERENCES}

1. Stinton $L$ and Shaffer $E$ (2012): Epidemiology of gallbladder disease: cholelithiasis and cancer. Gut Liver, 6(2): 172-187.

2. Shaffer E (2006): Epidemiology of gallbladder stone disease. Best Pract Res Clin Gastroenterol., 20(6): 981-996.

3. Litwin $D$ and Cahan $M$ (2008): Laparoscopic cholecystectomy. Surg Clin North Am., 88(6): 1295-1313.

4. Buanes $T$ and Mjaland $O$ (1996): Complications in laparoscopic and open cholecystectomy: a prospective comparative trial. Surg Laparosc Endosc., 6: 266-272.

5. Hollington $P$, Toogood $G$ and Padbury $R$ (1999): A prospective randomized trial of day-stay only versus overnight-stay laparoscopic cholecystectomy. Aust N Z J Surg., 69: 841-843.

6. Vivek MAKM, Augustine $A$ and Rao $R$ (2014): A comprehensive predictive scoring method for difficult laparoscopic cholecystectomy. J Minim Access Surg., 10: 62-67.

7. Liu C, Fan S, Lai E et al. (1996): Factors affecting conversion of 
laparoscopic cholecystectomy to open surgery. Arch Surg., 131(1): 98-101.

8. Yol S, Kartal A, Vatansev C et al. (2006): Sex as a factor in conversion from laparoscopic cholecystectomy to open surgery. JSLS., 10(3): 359-363.

9. Alqahtani R, Ghnnam W, Alqahtani M et al. (2015): Role of male gender in laparoscopic cholecystectomy outcome. Int J Surg Med., 1(2): 1-9.

10. Gabriel R, Kumar $S$ and Shrestha $A$ (1997): Evaluation of predictive factors for conversion of laparoscopic cholecystectomy. Kathmandu Univ Med J (KUMJ), 7: 26-30.

11. Younis $K$, Al-harbawi $L$ and Ashoor $O$ (2013): Evaluation of Clinical Parameters that Predict Difficulties During Laparoscopic Cholecystectomy, 12(2): 175-180.

12. Chang $\mathrm{W}$, Lee $\mathrm{K}$, Chuang $\mathrm{S}$ et al. (2006): The impact of prophylactic antibiotics on postoperative infection complication in elective laparoscopic cholecystectomy: a prospective randomized study. Am J Surg., 191(6): 721-725.
13. Ibrahim S, Hean T, Ho L et al. (2006): Risk factors for conversion to open surgery in patients undergoing laparoscopic cholecystectomy. World J Surg., 30: 1698-1704.

14. Baki A (2006): Pre-Operative Prediction of Difficult Laparoscopic Cholecystectomy Using Clinical and Ultrasono- Abstract: Patients and Methods, 27(3): 1-7.

15. Tosun A, Hancerliogullari KO, Serifoglu I et al. (2015): Role of preoperative sonography in predicting conversion from laparoscopic cholecystectomy to open surgery. European Journal of Radiology, 84(3): 346-9.

16. Akyurek N, Salman B, Irkorucu O et al. (2005): Laparoscopic cholecystectomy in patients with previous abdominal surgery. JSLS., 9(2): 178-183.

17. Sharma M (2014): Biliary Tract Anatomy and its Relationship with Venous Drainage. J Clin Exp Hepatol., 4(1): S18-S26. 